\title{
P02-026 - Model-based characterization of the PKPD relationship for canakinumab in CAPS: a step towards personalized
}

\author{
A Gautier, P Lowe, A Skerjanec, P McKernan, O Luttringer, M Fink \\ From 7th Congress of International Society of Systemic Auto-Inflammatory Diseases (ISSAID) \\ Lausanne, Switerland. 22-26 May 2013
}

\section{Introduction}

Canakinumab is a high-affinity fully human monoclonal antibody of the IgG1/k isotype, designed to bind and functionally neutralize the bioactivity of IL- $1 \beta$, which is recognized as one of the principal pro-inflammatory cytokines in cryopyrin associated periodic syndromes (CAPS).

\section{Objectives}

The objectives of the study were to describe the kinetics of canakinumab and dynamics of binding IL- $1 \beta$ in CAPS patients; to determine if these are different in 2- and 3 -year-old children versus older children and adults; and to explore the impact of CAPS phenotype (Muckle-Wells Syndrome [MWS], Familial Cold Autoinflammatory Syndrome [FCAS], Neonatal-Onset Multisystem Inflammatory Disease [NOMID]) on the kinetics of canakinumab and dynamics of binding to IL-1 $\beta$.

\section{Methods}

A pharmacokinetics (PK)-binding model was used to describe the kinetic and binding parameters of canakinumab and IL- $1 \beta$ in CAPS patients, and in other populations relative to CAPS. The subgroup of 7 CAPS patients who were 2 and 3 years of age at baseline was also compared to the overall CAPS population.

\section{Results}

The 7 CAPS patients did not show any difference in terms of PK. However, they showed a higher IL-1 $\beta$ turnover including IL- $1 \beta$ clearance and production. IL- $1 \beta$ levels were linked with the severity of the CAPS phenotype. In the pediatric population, MWS and especially NOMID patients had higher concentrations of the inert
canakinumab/IL-1 $\beta$ complexes after administration of canakinumab, indicating more cytokine in the body to be captured.

\section{Conclusion}

Correlation with clinical responses suggested that these increased levels of IL-1 $\beta$ may explain why younger and NOMID phenotype patients require higher doses or escalation to higher doses.

\section{Disclosure of interest}

A. Gautier Shareholder of: Novatis Pharma AG, Employee of: Novatis Pharma AG, P. Lowe Shareholder of: Novartis Pharma AG, Employee of: Novartis Pharma AG, A. Skerjanec Employee of: Novartis Pharma AG, P. McKernan Shareholder of: Novartis Pharma AG, Employee of: Novartis Pharma AG, O. Luttringer Shareholder of: Novartis Pharma AG, Employee of: Novartis Pharma AG, M. Fink: None Declared

Published: 8 November 2013

doi:10.1186/1546-0096-11-S1-A133

Cite this article as: Gautier et al:: P02-026 - Model-based characterization of the PKPD relationship for canakinumab in CAPS: a step towards personalized. Pediatric Rheumatology 2013 11(Suppl 1):A133. 